\title{
A tale of two shocks in SN 2004dj
}

\author{
Alak Ray ${ }^{1}$, Sayan Chakraborti ${ }^{2}$, Naveen Yadav ${ }^{1}$, Randall Smith $^{2}$, \\ Poonam Chandra ${ }^{3}$ and David Pooley ${ }^{4}$ \\ ${ }^{1}$ Tata Institute of Fundamental Research, Mumbai 400005, India \\ ${ }^{2}$ Harvard-Smithsonian Center for Astrophysics, Cambridge, MA 02138, USA \\ ${ }^{3}$ National Centre for Radio Astrophysics, TIFR, Pune 411007, India \\ ${ }^{4}$ Sam Houston State University, Huntsville, TX 77341, USA
}

\begin{abstract}
Type IIP SNe constitute a major fraction of all core-collapse supernovae and arise from massive stars that end their lives close to Red Supergiants. The blastwave from the SN interacting with the progenitor's circumstellar matter produces a hot region bounded by a forward and a reverse shock from which most of the X-ray emission originates. Analysis of archival Chandra observations of SN 2004dj, one of the nearest supernovae since SN 1987A, together with published data from radio and optical bands determines the pre-explosion massloss rate, blastwave speed, electron acceleration and magnetic field amplification efficiencies. Xray emission arises from both inverse Compton scattering by non-thermal electrons accelerated in the forward shock and from thermal emission from the supernova ejecta hit by the reverse shock. Determination of the properties of the radiating plasma based on the separation of thermal and non-thermal radiation differentiates different types of supernovae and their environments.
\end{abstract}

Keywords. supernovae, X-rays: general, radio continuum: general, circumstellar matter

\section{Introduction}

An important aspect of understanding of supernovae ( $\mathrm{SNe}$ ) relates to the kind of progenitor star they arise from and the circumstellar medium they explode into. The most common type of supernovae in a volume limited sample is Type IIP SNe (Smith et al. 2011). They are shown to arise from red supergiants (RSG) (Smartt et al. 2009 and references therein) with large Hydrogen envelope and a main sequence mass $\sim 8-18 M_{\odot}$. When their pre-explosion images are compared to those several years post-explosion they reveal the disappearance of the original RSG progenitor star (Maund and Smartt 2009). However the location of the progenitor stars on the H-R diagram, in some cases, shows that these stars are not at the predicted end points for single star tracks computed with evolutionary codes, and IIP SN 2008cn, for example, exploded as a yellow supergiant (YSG) rather than a RSG (Maund et al. 2011). Thus both progenitor and its environment in these explosions are complex and require probes, such as when the shock launched from the explosion interacts with the circumstellar medium (CSM) and radio and Xray emission generated in the hot, shocked region. The shock accelerates particles to high energies which provide us through radio and X-ray measurements such tools (see e.g. Chevalier et al. 2006). Usually, radio and X-ray emission from IIP SNe are rather faint, but if detailed spectral data could be obtained for a few X-ray bright SNe, they could provide a discrimination of thermal and nonthermal parts and constrain physical nature of the shock interaction. Due to the long lasting, bright (optical) light during the plateau stage, shock accelerated electrons that emit radio waves are likely to be subject to energy loss that affects the radio light curves and at the same time generate signals as nonthermal X-rays via Inverse Compton scattering (IC). SN 2004dj was one such nearby 

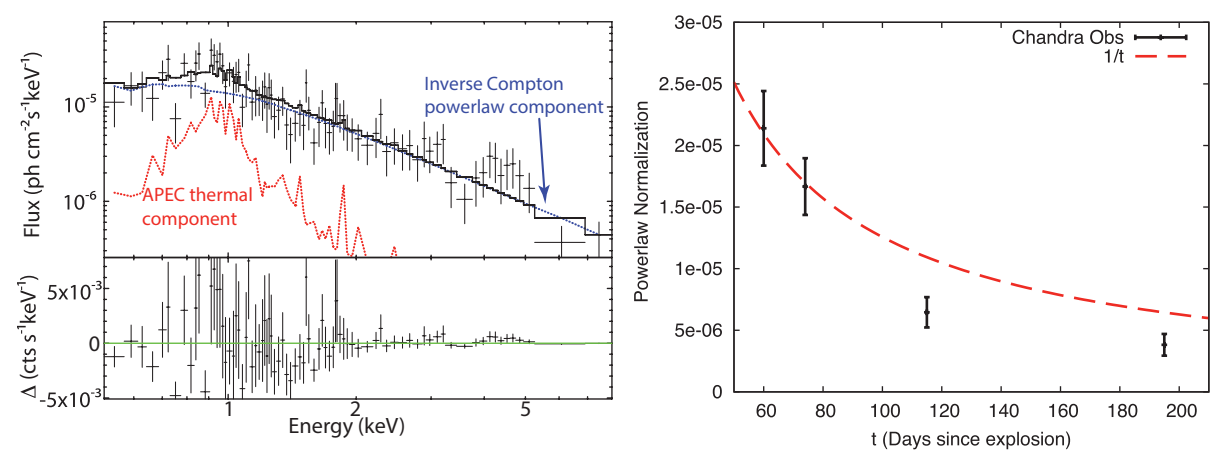

Figure 1. a) X-ray spectrum of SN 2004dj on 2004 August 9. Bars are counts from Chandra, dotted line is the power-law model for IC, dashed line is APEC model for the thermal plasma, while the solid line is the full model. The full model is dominated by the non-thermal flux even at $\sim 1 \mathrm{keV}$; b) Time evolution of the normalization of the non-thermal IC component obtained from simultaneous fits to Chandra spectra of SN 2004dj. The time interval is measured with respect to the explosion date of June 11 from Zhang et al. 2006. Line is best fit $\propto t^{-1}$ function.

$\mathrm{SN}(d=3.06 \mathrm{Mpc})$ which was extensively followed up in optical, radio and X-ray bands and here we discuss the X-ray lightcurve and spectral evolution. Together with radio data (arising from power law electrons with index p with $N(E) \propto E^{-p}$ ) they constrain the properties of the shocked plasma responsible for thermal and nonthermal emission, and determine the pre-SN mass loss rate (Chakraborti et al. 2012).

\section{SN 2004dj: X-ray and radio observations}

SN 2004dj was observed as a Target of Opportunity and detected by Chandra Xray Observatory on 2004 August 09 (Pooley and Lewin 2004). Chandra observed it for another three epochs during 2004 with ACIS-S (without gratings) for $50 \mathrm{ks}$ each time. The X-ray flux (0.5-8 keV) ranged from $8.8 \times 10^{-14} \mathrm{erg} \mathrm{cm}^{-2} \mathrm{~s}^{-1}$ to $2.0 \times 10^{-14} \mathrm{erg} \mathrm{cm}^{-2} \mathrm{~s}^{-1}$ between Aug 9 and Dec 22 (Chakraborti et al. 2012). Similarly, extensive observations of this SN took place with GMRT, MERLIN and the VLA (see Chandra et al. 2013).

The SN spectra was extracted into XSPEC 12.7.1 for further analysis. Data from each epoch of observation were jointly fitted with a combination of power law spectrum and collisionally ionized diffuse gas using APEC models (Smith et al. 2001) that is subject to photoelectric absorption column (wabs), see Fig 1a. We kept the same column density for wabs, plasma temperature for APEC, and photon index for power law at all epochs. The emission measure for APEC and the normalization of the power law were determined separately for each epoch (best fit $\left.N_{H}=(1.7 \pm 0.5) \times 10^{21} \mathrm{~cm}^{-2}\right)$.

Chandra observations of SN 2004dj indicate that the IC component of the X-ray flux falls with time. The spectrum of X-rays also softens with time. The quality of the SN 2004dj spectra allows a quantitative explanation for the first time. Although both thermal and non-thermal components decrease eventually, their proportion changes with time. At early times the spectra is dominated by the inverse Compton flux and is therefore harder. As the supernova ages, the source of seed optical photons to be scattered turns off. So at late times, the spectra is dominated by emission from the reverse-shocked thermal plasma and is softer.

The blastwave shock is the site of acceleration of particles, including electrons, to relativistic speeds. These electrons emit radio radiation via synchrotron emission in the magnetic field which is in turn amplified by the shock. The simplest model by Chevalier 1982, is to assume that a fraction $\epsilon_{e}$ or $\epsilon_{B}$ of the thermal energy is used to accelerate electrons 

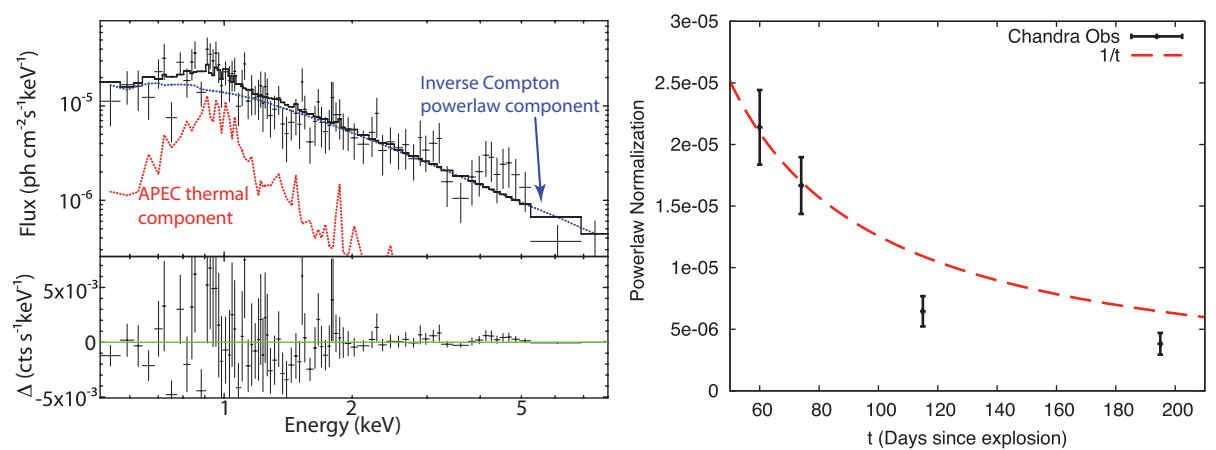

Figure 2. a) Normalizations of thermal (APEC model) and non-thermal (power-law model) fluxes for SN 2004dj on 2004Aug09; b) Chandra data breaks the degeneracy between the two components and determine their temperature and the photon index.

and amplify magnetic fields respectively. The radio emission from a supernova is dependent upon these fractions. According to Chevalier et al. 2006, the radio emission measures $S_{\star}=A_{\star} \epsilon_{B-1} \alpha^{8 / 19}=1.0(f / 0.5)^{-8 / 19}\left(F_{\mathrm{p}} /(\mathrm{mJy})\right)^{-4 / 19}(\mathrm{D} /(\mathrm{Mpc}))^{-8 / 19}(\nu / 5 \mathrm{GHz})^{-4 / 19} \mathrm{t}_{10}^{2}$ where $F_{\mathrm{p}}$ is the peak flux at peak frequency $\nu$ at $10 \times t_{10}$ days from the explosion and $\epsilon_{B-1}=\epsilon_{B} / 0.1$. The equipartition factor is defined as $\alpha \equiv \epsilon_{e} / \epsilon_{B}$. Using the $4.99 \mathrm{GHz}$, radio light curve from Beswick et al. 2005 for SN 2004dj, we have $S_{\star}=5.1$. These relativistic electrons also contribute to the X-ray flux through IC scattering of optical photons. For an electron index $\mathrm{p}=3$ this is expected to generate a power law in X-rays with photon index 2 , consistent with the observations. The normalization of the inverse Compton flux obtained for the first epoch $\left(t_{10} \sim 6\right)$ of Chandra observations, is found to be $(3.8 \pm 0.5) \times 10^{37} \mathrm{ergs} \mathrm{s}^{-1}$ (see Fig 2a). This together with $S_{\star}=5.1$ as found in this work, $V_{4}=0.9$ as implied by the temperature of the reverse-shocked plasma (Fig $2 \mathrm{~b}$ ), $L_{\mathrm{bol}}=0.89 \times 10^{42} \mathrm{erg} \mathrm{s}^{-1}$ from Zhang et al. 2006 , we get $\alpha \sim 23 \times \gamma_{\text {min }}^{-19 / 11}$. We take $\gamma_{\min } \sim 2.5$ as used by Chevalier and Fransson 2006 for SN 2002ap, which then implies $\alpha \sim 4$.8. Since we know $S_{\star}$ from radio synchrotron, $A_{\star}$ from thermal X-rays and $\alpha$ from inverse Compton, we can use the definition of $S_{\star}$ to get $\epsilon_{B} \equiv 0.1 \times \epsilon_{B-1} \sim 0.082$, which in turn implies $\epsilon_{e} \equiv \alpha \times \epsilon_{B} \sim 0.39$. Hence both $\epsilon_{e}$ and $\epsilon_{B}$ are determined independently.

\section{Implications}

The time variation of the IC flux is due to the expansion of the blastwave and the fading away of the supernova's supply of the seed photons. The normalization of the IC flux scales as (Chevalier et al. 2006): $E \times d L_{\mathrm{IC}} / d E \propto L_{\mathrm{bol}}(t) / t$. Therefore during the plateau phase of the optical light curve of the SN where the bolometric luminosity $L_{b o l}$ is nearly constant, the IC flux is expected to fall off as $\propto t^{-1}$. At late times, since $L_{b o l}$ itself would be decreasing, the IC flux would deviate from the $t^{-1}$ dependence. In Fig $1 \mathrm{~b}$ this prediction (shown by the dashed line) is consistent with the observed X-ray flux components of SN 2004dj. The emission measure from the thermal part of the X-ray flux (from the reverse shocked ejecta) can be related to the mass loss parameter $\dot{M} / v_{W}$ if we can determine the blastwave shock radius from the velocity (e.g. with the postshock temperature or from radio broadband spectra assumed to be a synchrotron self absorbed spectrum). The APEC fit, along with distance of $3.06 \mathrm{Mpc}$ and the emission measure gives: $\dot{M}=(3.2 \pm 1.1) \times 10^{-7}\left(v_{\mathrm{w}} / 10 \mathrm{~km} / \mathrm{s}\right) \mathrm{M}_{\odot} \mathrm{yr}^{-1}$ This mass loss rate is however substantially less than that predicted empirically for RGB branch following de Jager et al. 1988, for an estimated progenitor mass of $\sim 15 M_{\odot}$ (Maiz-Applellaniz et al. 2004). 
Around a third of the energy thermalized by the collision of the ejecta with circumstellar matter is processed by the shock to accelerate electrons to relativistic velocities. Another tenth of the thermal energy available is used in the turbulent amplification of magnetic fields. Electrons can "cool" via Inverse Compton cooling against low-energy photons from the SN photosphere. The cooling break frequency in the radio bands can be obtained by demanding that the IC loss time scale is equal to the age of the SN. For the parameters relevant to $\mathrm{SN} 2004 \mathrm{dj}$, the predicted $\nu_{\mathrm{IC}}$ is within the coverage of the VLA band for about 180 days which should be seen in its radio spectral index variations.

\section{Conclusions}

For bright IIP SN like 2004dj we can separate the thermal component and the IC components and determine the pre-explosion mass-loss rate, electron acceleration and magnetic field amplification efficiencies. Another IIP SN 2011ja X-ray brightened between two Chandra observations (Chakraborti et al. 2013). This may imply a complex circumstellar medium, e.g. an explosion inside a rarefied cavity surrounded by a denser medium characteristic of an older and slower Red Supergiant wind, like SN 1987A. Progenitor environments of IIP SNe may be more complex than what has been interpreted so far. Radio observations of another IIP SN 2012aw (Yadav et al. 2013) show evidence of electron cooling during the (optical light curve) plateau phase.

\section{References}

Beswick, R. J., et al. 2005, ApJ Letters, 623, L21

Chakraborti, S., et al. 2013, these Proceedings \& arXiv: 1302.7067

Chakraborti, S., Yadav, N., \& Ray, A., et al. 2012, ApJ, 761, 100

Chandra, P., et al. 2013, In preparation

Chevalier, R. A. 1982, ApJ, 258, 790

Chevalier, R. A., Fransson, C., \& Nymark, T. K. 2006, ApJ, 641, 1029

Chevalier, R. A. \& Fransson, C. 2006, ApJ, 651, 381

de Jager, C., Nieuwenhuijzen, H., \& van der Hucht, K. A. 1988, Astron. Astroph. Suppl., 72, 259

Maiz-Apellaniz, J., et al. 2004, ApJ Letters, 615, L113

Maund, J., et al. 2011, ApJ Letters 739, L37

Maund, J. \& Smartt, S. 2009, Science 324, 486

Pooley, D. \& Lewin, W. H. G. 2004, IAUC, 8390, 1

Smartt, S., et al. 2009, MNRAS 395, 1409

Smith, N., et al. 2011, MNRAS, 412, 1522

Smith, R. K., et al. 2001, ApJ Letters, 556, L91

Yadav, N., et al. 2013, these Proceedings

Zhang, T., et al. 2006, AJ 131, 2245

\section{Discussion}

SAHA, LAB: Is there any time variability in flux for SN2004dj. Due to presence of reverse shocks, there could be turbulence in the SN region. So there could be magnetic amplification. Did you see any flux enhancement due to the reason mentioned above?

RAY: The X-ray flux (0.5-8 keV band) decreases with time. Radio emission is the characteristic indicator of synchrotron radiation in a magnetic field amplified near the forward shock although electrons accelerated at the reverse shock may contribute a minor fraction of the total flux density. The flux density at $5 \mathrm{GHz}$ decreases initially. 